\title{
AVALIAÇÃO POR CFD DA INFLUÊNCIA DO MODELO DE BALANÇO POPULACIONAL NA DISPERSÃO DE LÍQUIDO EM UM LAVADOR VENTURI
}

\section{CFD EVALUATION OF THE INFLUENCE OF POPULATION BALANCE EQUATION ON LIQUID DISPERSION IN A VENTURI SCRUBBER}

\author{
L. M. MARINI ${ }^{1}$, A. S. SOUZA ${ }^{1}$, V. G. G. BÉTTEGA ${ }^{1}$ e R. BÉTTEGA ${ }^{1, *}$ \\ ${ }^{1}$ Federal University of São Carlos, Department of Chemical Engineering, São Carlos, São Paulo, Brazil
}

${ }^{*}$ Corresponding author. Federal University of São Carlos, Department of Chemical Engineering, São Carlos, São Paulo, Brazil, Phone: +55 1633518440 e-mail addressl: bettega@ufscar.br (R. Béttega).

\begin{tabular}{l} 
A R T I C L E I N F O \\
\hline Article history: \\
Received 2020-06-29 \\
Accepted 2020-09-04 \\
Available online 2020-09-04 \\
$p$ a l $a v r a s-c h a v e$ \\
fluidodinâmica computacional \\
tamanho de gota \\
modelo de balanço populacional \\
$k$ e y $w$ o $d s$ \\
computational fluid dynamics \\
droplet size \\
population balance equation
\end{tabular}

A B S T R A C T

In this work the influence of including the Population Balance Equation (PBE) in Computational Fluid Dynamics (CFD) simulations of a Venturi Scrubber was performed in order to analyze the dispersion of liquid injected in the equipment. The results of simulations with and without the PBE were compared, and the inclusion of the effects of the models of droplet breakage and coalescence were analyzed. The results showed that the inclusion of PBE had little effect on the pressure drop in the Venturi Scrubber. The contours of volume fraction of the droplets showed that the inclusion of PBE allowed to evaluate the variation of droplet diameter and its dispersion in the equipment. CFD simulations using the PBE can contribute to the design of Venturi Srubbers in order to verify the dispersion of injected fluid provided by the corresponding geometry.

R E S UM O

Neste trabalho foi analisada a influência da inclusão do Modelo de Balanço Populacional (MBP) em simulações por Fluidodinâmica Computacional (CFD) na dispersão do líquido injetado em um Lavador Venturi. Foram comparados os resultados de simulações com e sem a inclusão do MBP, bem como avaliada a inclusão dos efeitos dos modelos de quebra e coalescência de gotas. Os resultados mostraram que a inclusão do MBP apresentou efeito pouco pronunciado sobre a queda de pressão no Lavador Venturi. Nos contornos de fração volumétrica das gotas, foi observado que a inclusão do MBP possibilitou avaliar a variação de diâmetro de gota e a sua dispersão no equipamento. Simulações por CFD utilizando o MBP podem contribuir para projetos de Lavadores Venturi, de modo a verificar a dispersão de líquido injetado que a geometria correspondente proporciona. 


\section{INTRODUÇÃO}

Em virtude das legislações mais rigorosas e do aumento da preocupação com o meio ambiente, as indústrias têm apresentado uma particular atenção às emissões de gases poluentes e material particulado. Um equipamento bastante utilizado na indústria para o controle da poluição do ar é o lavador de gases, cuja aplicação é tanto para o controle da emissão de gases quanto para coleta de partículas, como por exemplo a fuligem (Silva, 2009).

Dentre os diversos tipos de lavadores industriais, o lavador Venturi se destaca por ser um dos mais eficientes na coleta de partículas submicrométricas. O equipamento é constituído por três partes: seção convergente, garganta (constrição) e seção divergente. Durante o seu funcionamento, o líquido de lavagem do gás é geralmente introduzido por bocais ou orifícios localizados na garganta. Ao entrar em contato com o gás em alta velocidade, o líquido se rompe em inúmeras gotas que farão a coleta das partículas (Guerra et al. 2012).

A eficiência de coleta das partículas depende da uniformidade e da distribuição de tamanho das gotas ao longo do equipamento (Viswanathan, 1997). As condições operacionais e de projeto do lavador influenciam na uniformidade na dispersão do líquido (Guerra et al., 2017). Por sua vez, a distribuição de tamanhos de gotas aborda fenômenos mais complexos, tais como a ruptura e a coalescência das gotas, cuja ocorrência altera sua dispersão e distribuição ao longo do equipamento. Assim, modelos para previsão da dispersão de líquido que considerem esses fenômenos são importantes para o projeto de lavadores Venturi, bem como para otimizar suas condições operacionais e garantir uma melhor eficiência de coleta.

Neste contexto, utilizar técnicas de simulação, tais como a fluidodinâmica computacional (CFD), é uma alternativa interessante para o projeto e estudo dos lavadores Venturi, diminuindo o custo final, economizando tempo útil $\mathrm{e}$ possibilitando a observação mais detalhada do escoamento quando comparada a modelos matemáticos simplificados. Assim sendo, o objetivo deste trabalho foi avaliar a influência da implementação do Modelo de Balanço Populacional, com e sem a inclusão da quebra e coalescência de gotas, nas respostas das simulações CFD de um lavador Venturi. Para isso, foram analisados o perfil de frações volumétricas de água e a queda de pressão no equipamento aplicando os diferentes modelos.

\section{MATERIAIS E MÉTODOS}

A configuração do lavador Venturi simulado e as condições de operação utilizadas tiveram como base o trabalho de Guerra et al. (2012). O equipamento possui seção quadrada, com orifícios para injeção de fluido sobrepostos de 0,$7 ; 1,0$ e 1,3 $\mathrm{mm}$ de diâmetro posicionados na entrada da garganta.

As características principais do modelo aplicado, bem como procedimento numérico de solução são apresentados na Tabela 1. A injeção de líquido foi realizada por meio da inclusão de pontos de injeção no domínio computacional, representando bicos injetores de fluido com $1 \mathrm{~mm}$ de diâmetro. A solução das equações da continuidade, da quantidade de movimento e referentes aos modelos multifásicos aplicados foi feita pelo método dos volumes finitos por meio do software ANSYS FLUENT 18.1. As simulações foram realizadas em um computador ACER ${ }^{\circledR}$ NITRO AN515-51 Intel® COREE i7$7700 \mathrm{HQ}$.

Tabela 1 - Modelos matemáticos e parâmetros numéricos utilizados nas simulações.

\begin{tabular}{|c|c|}
\hline $\begin{array}{l}\text { Condições de } \\
\text { Simulação }\end{array}$ & Descrição \\
\hline $\begin{array}{l}\text { Modelos } \\
\text { multifásicos } \\
\text { Modelo de } \\
\text { turbulência }\end{array}$ & $\begin{array}{l}\text { Euleriano ï VOF (Volume of Fluid) e } \\
\text { mistura } \\
\text { k-ŮRNG }\end{array}$ \\
\hline $\begin{array}{l}\text { Condições de } \\
\text { contorno }\end{array}$ & $\begin{array}{l}\text { Discreto (com agregação e quebra de } \\
\text { núcleos) - (Hagesather et al., 2002) } \\
\text { - Entrada de líquido no bico inferior: } \\
\text { velocidade prescrita, } 9,7 \mathrm{~m} / \mathrm{s} \\
\text { - Entrada de líquido no bico superior: } \\
\text { velocidade prescrita, } 9,7 \mathrm{~m} / \mathrm{s} \\
\text { - Entrada de Ar: velocidade prescrita, } 23 \\
\text { m/s } \\
\text { - Parede: Condição de não-deslizamento } \\
\text { para ambas as fases } \\
\text { - Saída: Pressão atmosférica }\end{array}$ \\
\hline $\begin{array}{l}\text { Acoplamento } \\
\text { pressão- } \\
\text { velocidade }\end{array}$ & PISO \\
\hline $\begin{array}{l}\text { Discretização } \\
\text { das equações }\end{array}$ & $\begin{array}{l}\text { Upwind de primeira ordem para } \\
\text { Momentum, fração volumétrica, } \\
\text { turbulência, e discretização espacial do Bin }\end{array}$ \\
\hline $\begin{array}{l}\text { Parâmetros } \\
\text { de relaxação }\end{array}$ & 0,1 a 0,4 \\
\hline
\end{tabular}

A malha computacional foi construída no software GAMBIT 2.4.6. O teste de independência de malha foi feito conforme o método GCI (Grid Convergence Index), desenvolvido por Roache (1994). Três diferentes configurações de malha $(336.879,741.314$ e 1.637 .200 elementos) foram testadas e aquela que apresentou desvio aceitável pelo GCI, bem como tempo de simulação adequado, foi escolhida. Assim, foi utilizada uma malha computacional com 741.314 células, conforme ilustrado na Figura 1.

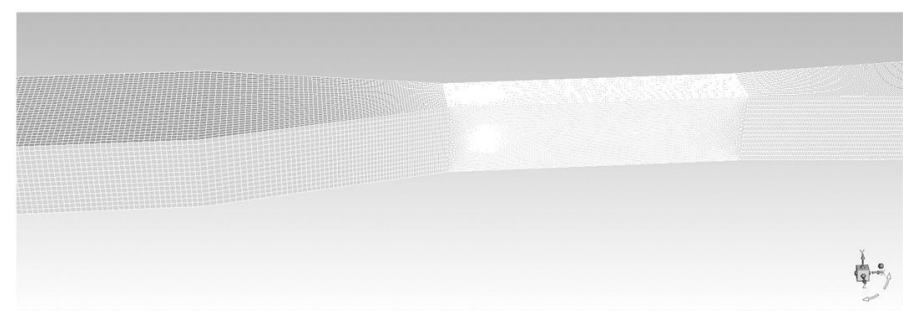

Figura 1 - Malha Computacional.

Considerando o modelo e o procedimento numérico proposto, os casos avaliados são apresentados na Tabela 2. Os chamados bins são classes de diâmetro que as gotas podem adotar durante a simulação, dependendo da situação de tensão em que ela se encontra. Os bins são inseridos pelo usuário nas condições de contorno, ou seja, caso sejam inseridos 6 (seis) 
bins, a gota pode adotar até 6 diferentes diâmetros. Entretanto, caso somente haja somente um único bin, a gota permanecerá somente com o mesmo diâmetro durante toda a sua trajetória no equipamento. Para os casos de 1 bin, foi injetado somente uma fração volumétrica de líquido igual a 1. Já para o caso de 6 bins, as frações volumétricas de líquido injetadas para bin $0,1,2,3$, 4 e 5 foram, respectivamente, $0,0,65,0,33,0,015,0,005$ e 0 , as quais correspondem aos diâmetros de gota de $1,000 \times 10^{-3} \mathrm{~m}$, $1,933 \times 10^{-4} \mathrm{~m}, 3,737 \times 10^{-5} \mathrm{~m}, 7,225 \times 10^{-6} \mathrm{~m}, 1,397 \times 10^{-6} \mathrm{~m} \mathrm{e}$ $2,700 \times 10^{-7} \mathrm{~m}$.

Tabela 2 - Casos estudados.

\begin{tabular}{ccc}
\hline Caso & Modelo multifásico e MBP & Bins \\
Caso 1 & VOF (sem MBP) & - \\
Caso 1.1 & Mistura (sem MBP) & - \\
Caso 1P & Mistura (com MBP) & 1 bin \\
Caso 1PQ & Mistura (com MBP, Quebra) & 1 bin \\
Caso 1PQC & Mistura (com MBP, Quebra, & 1 bin \\
Caso 6P & Coalescência) & 6 bins \\
\hline
\end{tabular}

Os casos foram comparados com base nos contornos de fração volumétrica dos bins, exibidos a partir de um plano posicionado na seção transversal do Venturi. Foram monitorados os valores de fração volumétrica de água multiplicados pela fração média local de cada bin, considerando uma escala entre 0 (zero) a 0,001 para cada um dos 6 (seis) bins aplicados, sabendo que os bins são enumerados de modo decrescente em tamanho (bin- $0>$ bin- $1>$ bin- $2>$ bin- $3>$ bin- 4 $>$ bin-5).

\section{RESULTADOS}

\subsection{Queda de pressão total}

Os resultados obtidos para a queda de pressão entre a entrada e a saída do equipamento, são apresentados na Tabela 3. Foi observada uma discreta variação nos valores de queda de pressão quando houve alteração no modelo multifásico de VOF para o de mistura. No entanto, incorporar o MBP no modelo Euleriano de mistura, bem como adicionar os diferentes fenômenos de quebra, coalescência e distribuição de tamanhos de gota, não proporcionou diferença substancial na queda de pressão para essa geometria de Lavador Venturi.

Tabela 3 - Valores de queda de pressão para cada caso simulado.

\begin{tabular}{cc} 
Caso & $\boldsymbol{\Delta P}[\mathbf{P a}]$ \\
Caso 1 & 1388,74 \\
Caso 1.1 & 1314,91 \\
Caso 1P & 1315,00 \\
Caso 1PQ & 1315,00 \\
Caso 1PQC & 1315,00 \\
Caso 6P & 1314,96 \\
\hline
\end{tabular}

\subsection{Distribuição de concentração}

Nas Figuras 2 e 3 são apresentados os contornos de distribuição de fração volumétrica de fase considerando os diferentes casos simulados. A alteração no número de bins da simulação proporcionou a resposta apresentada na Figura 2.

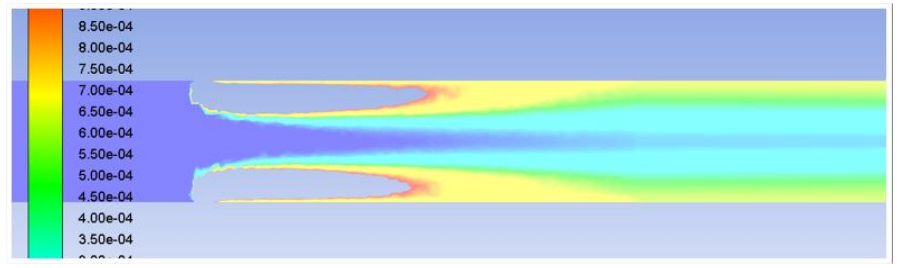

Figura 2 - Perfil de fração de bin-2 (escala de 0 a 0,001 ) da simulação 1P, sendo esta obtida pela multiplicação da fração volumétrica de água pela opção "bin-2-fraction".

Na Figura 4 é ilustrada a influência do modelo de Quebra e Coalescência, para o qual entraram somente gotas de bin-2 pelos jatos.

Os resultados demonstraram que o modelo de balanço populacional possibilitou avaliar a localização da concentração de gotas de determinado diâmetro no interior do equipamento, para verificar se a geometria possibilita uma boa dispersão do(s) fluido(s) injetado(s). Além da localização, a detecção de uma possível quebra ou coalescência pode ser prevista.
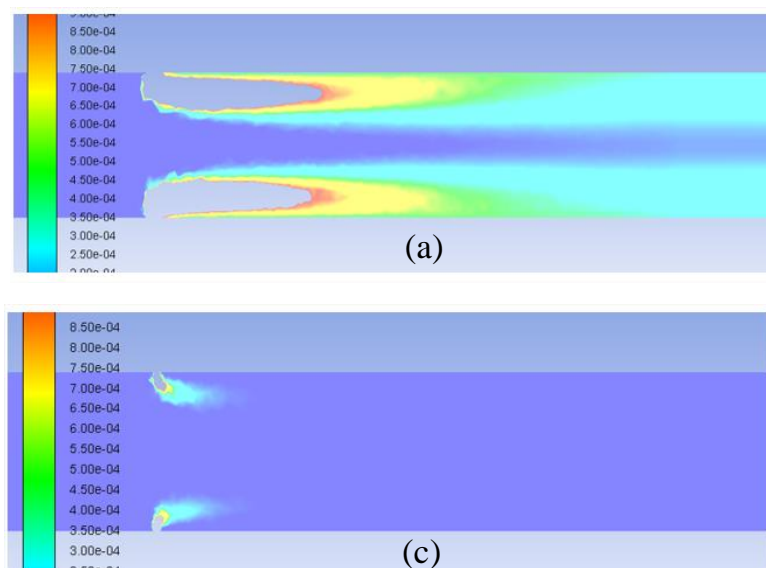
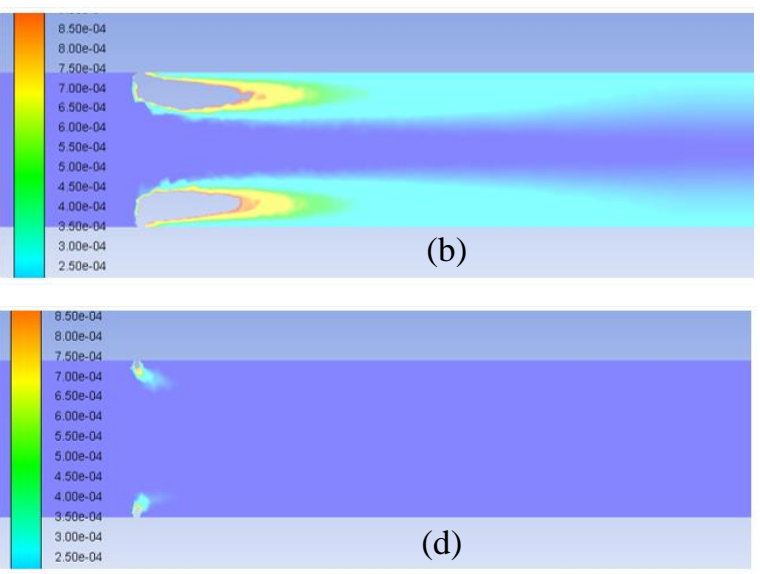

Figura 3 - Perfil de fração volumétrica de a) bin-1, b) bin-2, c) bin-3, d) bin-4, em escala de 0 a 0,001, da simulação 6P, sendo esta obtida pela multiplicação da fração volumétrica de água pela opção "bin-i-fraction". 

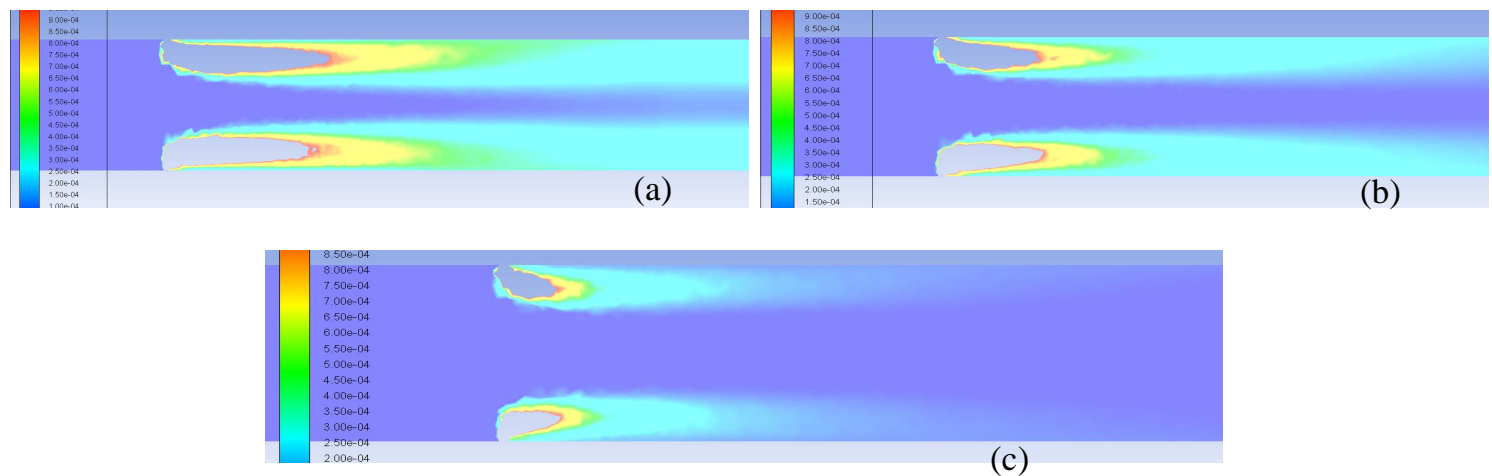

Figura 4 - Perfil de fração volumétrica de a) bin-0, b) bin-1, c) bin-2, em escala de 0 a 0,001 para o caso 1PQC, sendo esta obtida pela multiplicação da fração volumétrica de água pela opção "bin-i-fraction".

\section{CONCLUSÃO}

A aplicação do Modelo de Balanço Populacional em simulações CFD de um lavador Venturi foi avaliada para diferentes casos. Pouca influência da inclusão do balanço populacional foi observada sobre a resposta simulada para a queda de pressão. No entanto, a inclusão do balanço populacional foi importante para descrever a distribuição de gotas no equipamento, bem como para prever a possível quebra e coalescência entre elas. $\mathrm{O}$ balanço populacional apresentou-se como um importante modelo a ser incorporado nas simulações CFD para a análise e projeto de Lavadores Venturi.

\section{AGRADECIMENTOS}

O presente trabalho foi realizado com apoio da Coordenação de Aperfeiçoamento de Pessoal de Nível Superior ï Brasil (CAPES) ï Código de Financiamento 001. Os autores também agradecem ao CNPq ï Código de Financiamento [142102/2019-9], bem como pela bolsa de Iniciação Científica.

\section{R E F E R Ê N C I A S}

DAMIAN R. B. Acoplamento de Balanço Populacional à Simulação Computacional de Escoamentos Multifásicos Polidispersos. Dissertação (Mestrado em Engenharia Química) - Universidade Federal do Rio de Janeiro, Rio de Janeiro, RJ, 2007.

GUERRA V. G., ACHILES A. E., BETTEGA R.; Influence of Droplet Size Distribution on Liquid Dispersion in a Venturi Scrubber: Experimental Measurements and CFD Simulation. Industrial and Engineering Chemistry
Research, v. 56, n. 8, p. 2177-2187, 2017.

GUERRA, V. G.; BÉTTEGA, R.; GONÇALVES, J.A.S.; COURY, J.R. Pressure Drop and Liquid Distribution in a Venturi Scrubber: Experimental Data and CFD Simulation, Industrial and Engineering Chemistry Research, v.51, p. 8049-8060, 2012.

HAGESAETHER L., JAKOBSEN H.A.1, and SVENDSEN H.F. ñA Model for Turbulent Binary Breakup of Dispersed Fluid Particlesò. Chemical Engineering Science. v. 57, n. 16. p. 3251ї 3267. 2002.

HONDA V. K., BÉTTEGA R., GUERRA V. G.; Avaliação da Hidrodinâmica de um Lavador Venturi: Dados Experimentais e Simulação Numérica. Anais do XXXVII Congresso Brasileiro de Sistemas Particulados - ENEMP, 2015.

QUEDEVILLE V., OUAZAITE H., POLIZZI B., FOX R.O., VILLEDIEU P., FEDE P., LÉTISSE F., MORCHAIN J.; A two-dimensional population balance model for cell growth including multiple uptake systems. Chemical Engineering Research and Design, 2018.

ROACHE, P. J. (1994). Perspective: A Method for Uniform Reporting of Grid Refinement Studies. Journal of Fluids Engineering, 116, $405 \mathrm{ï} 413$.

SILVA D. A. L. Lavadores Venturi Na Coleta De Particulados Finos Emitidos Com A Queima De Resíduos Madeiráveis: Influência Das Principais Variáveis De Processo Na Eficiência Fracionária Para Partículas De 0 A $10 \mu \mathrm{m}$. Universidade Estadual Paulista ñJúlio De Mesquita Filhoò, Itapeva, São Paulo, 2009.

VISWANATHAN, S. Modeling of Venturi scrubber performance. Industrial and Engineering Chemistry Research, v.36, n.10, p.4308-4317, 1997. 